\title{
HACIA UNA ONTOLOGÍA POLÍTICA POSMETAFÍSICA. POLÍTICA Y ESCRITURA EN GEORGES BATAILLE
}

\author{
Juan José Martínez Olguín* \\ IDAES / UNSAM / CONICET \\ jjmartinezolguin@gmail.com
}

\begin{abstract}
RESUMEN / ABSTRACT
Con el objeto de identificar lo que creemos constituye una ontología política posmetafísica en el pensamiento de Bataille -fuertemente vinculada a la práctica de la escritura- el presente trabajo intenta situar la especificidad teórica que ocupa el exceso como problema ontológico en su filosofía, a partir del desarrollo de un recorrido que reflexiona sobre el estatuto de dicho exceso en Lévi-Strauss y el posestructuralismo, por un lado, y en el pensamiento político posfundacional, por el otro.
\end{abstract}

Palabras Clave: política, ontología, escritura, Bataille, posmetafísica.

\section{TOWARDS A POST METAPHYSICAL POLITICAL ONTOLOGY. POLITICS AND WRITING IN GEORGES BATAILLE}

In order to identify what we believe constitutes a post metaphysical political ontology in Bataille's thought - strongly linked to the practice of writing - this essay attempts to place the theoretical specificity that excess occupies as an ontological problem in its philosophy from developing a journey that reflects on the statute of such excess in Lévi-Strauss and post-structuralism, on the one hand, and in post-foundational political thinking, on the other.

KEYWORDS: Politics; ontology; writing; Bataille; post metaphysics.

\section{Introducción}

RA Hay un dato, que no solo pertenece al dominio de lo biográfico, que ilustra con toda precisión una de las marcas distintivas de la vida de Bataille: el gusto que el filósofo francés supo tener por los excesos y por las experiencias más lujuriosas y diversas. En la larga lista que bien podría describir esta marca distintiva de su vida -tan conocida como poco ocultada por el autor-habría que incluir sin dudas sus incursiones

Dr. en Filosofía por la Universidad de Paris VIII Vincennes -Saint Denis y Dr. en Cs. Sociales por la Universidad de Buenos Aires. Becario posdoctoral del CONICET con sede de trabajo en el Centro de Estudios Sociopolíticos del IDAES (UNSAM). Docente en la Universidad de Buenos Aires y autor de Politique de l'écriture (L'Harmattan, Paris, 2018). 
por la bebida, el juego y el sexo - la habitualidad con la que Bataille solía asistir a los burdeles es de hecho bien conocida-. Quizás la mejor definición a propósito de esta faceta de la vida de Bataille es la que Michel Leiris, amigo del propio Bataille y en su época subdirector del periódico surrealista Documents, del que Bataille también participó como secretario general, le transmitió a Michel Surya en la biografía que este último le dedicó al pensador francés: "Cuando conocí a Bataille éste llevaba ya la vida más disoluta. Era licenciado, bebedor y jugador” (Surya 2014, p. 33) ${ }^{1}$. Es cierto que, para ser justos, esta calificación de la vida de Bataille como una vida disoluta, es decir, como una vida entregada a los vicios y los placeres, no debería hacernos perder de vista que a pesar de la precisión que posee para indicar lo que estamos intentando remarcar no responde con toda fidelidad a los hechos de su biografía desde una perspectiva más general. Porque en sus años de juventud, el joven Bataille optó, luego de la muerte de su padre, por buscar en la fe y en la religión cristiana -en una vida más ascética, dicho en otras palabras- el motor de su vida. Una búsqueda que sin embargo fracasó: Bataille -escribe Surya en la biografía que citábamos- en aquellos años "era alguien que quiere creer, más que (..) alguien que realmente cree" (Surya 2014, p.87) ${ }^{2}$. En todo caso, entonces, esta vida disoluta y marcada por los excesos es la que distingue, para ser más precisos, al Bataille adulto. Ahora bien: lo que sí configura una certeza a propósito de este vínculo del estilo de vida de Bataille con los excesos es su relación, por demás estrecha, con su pensamiento o con su reflexión filosófica. Son pocos los autores cuyo pensamiento y cuya vida alcanzan una articulación tan profunda. Porque Bataille no solo vivió, desde la adultez hasta su muerte, una vida de excesos. No solo fue su vida la que estuvo marcada por los vicios y los excesos. Su pensamiento, lo más hondo de su pensamiento estuvo también marcado por el exceso pero, va de suyo, por el exceso como objeto de reflexión filosófica. El motivo del exceso ocupa en la trayectoria intelectual de Bataille un lugar central. El texto que, en este sentido, refleja con mayor claridad esta centralidad temática del exceso en su filosofía es probablemente su célebre ensayo La part maudite (Bataille 1967b). Escrito en 1949, Bataille distingue allí entre dos tipos o dos sentidos bien distintos de economía. En primer lugar-sostiene-, existe un sentido de lo que podemos llamar economía que es aquel que se corresponde o se identifica con el uso que normalmente hacemos del término economía y que refiere al modo mediante el cual los individuos producimos y hacemos uso de la riqueza. Pero este sentido de la palabra economía, escribe Bataille, es en realidad un sentido restringido. Porque vista con el cristal o a través de este punto de vista o perspectiva, como la esfera de la vida que da cuenta de la actividad a través de la cual los hombres producen y

1 Además de la biografía ya citada de Michel Surya, puede consultarse a este respecto la excelente tesis sobre Bataille de Marisol Pornofiello (2017), en donde la autora ahonda con mayor precisión sobre este aspecto de la vida de Bataille.

2 Esta búsqueda, para matizar un poco este "fracasó", en algún punto permaneció, pese a todo, en sus textos posteriores a propósito de su reflexión sobre el misticismo y lo sagrado. Puede verse, a este respecto, dos textos en particular: La experiencia interior (Bataille 2016) y los ensayos del autor compilados bajo el nombre de La conjuración sagrada (Bataille 2003). 
consumen $-y$, podríamos agregar, distribuyen- la riqueza, la economía solo responde a un aspecto particular de una actividad más amplia y mucho más abarcadora - de la que la primera, por lo tanto, forma parte- que es la actividad terrestre "considerada como un fenómeno cósmico" (Bataille 1967b, pp. 58-59. La traducción es mía). Este último sentido -al que Bataille llama general- de la palabra economía, el que remite a la actividad terrestre considerada como un fenómeno cósmico, alude ni más ni menos que al "movimiento en la superficie del globo que resulta del recorrido de la energía en este punto del universo" (Ibid. La traducción es mía). En este punto del universo, es decir en el planeta Tierra. La actividad económica de los hombres, la que refiere a la producción, al consumo y a la distribución de los bienes o la riqueza es así solo una parte, una instancia derivada de la economía en su sentido general: pues ésta "solo se apropia -escribe siempre Bataille en La part maudite-, de ese movimiento terrestre para ciertos fines: el del mantenimiento de la vida" (Ibid. La traducción es mía), es decir, de la vida de los hombres. Pero existe sin embargo un factor fundamental que determina por igual ambos tipos de economía - a la economía en su sentido restringido, y a la economía en su sentido general-. Hay un hecho elemental, puesto en otras palabras, que explica el modo en que ambas actividades se desenvuelven y condicionan mutuamente: el excedente de energía a partir del cual funcionan y son posibles: "En la superficie del globo -escribe Bataille- la energía está siempre en exceso (en excès)" (Bataille 1967b, p. 61. La traducción es mía). Se trata, en suma, de la "superabundancia" (surabondance) de energía solar que recibe la Tierra para el mantenimiento de la vida. La Tierra recibe siempre más energía de la necesaria para la reproducción de los organismos vivos que la habitan. Y este excedente de energía que recibe la Tierra condiciona y determina la actividad del hombre para el mantenimiento de su propia vida. La economía en su sentido restringido, por lo tanto, está también condicionada por este excedente de energía. Tanto la materia viva en general, la vida de los organismos vivos en su generalidad, como la materia viva que es el hombre, la vida humana, están atravesados por este excedente suplementario de energía que las constituye como tales, que las constituye y, por supuesto, las desborda porque las excede. Como vemos, el exceso ocupa un lugar central y preponderante en la filosofía de Bataille. Forma parte de su pensamiento pero -decíamos también- de lo más profundo de su pensamiento o de su reflexión teórica. Para Bataille, para decirlo de otro modo, el exceso no es de ningún modo un aspecto accidental de su teoría. Y no lo es en un doble sentido. Porque el tema lo ocupa y preocupa en varios textos -ya no se trata, pues, solo y únicamente de $L a$ part maudite: el tema vuelve en ensayos como La notion de dépense (Bataille, 1967a) o en el prefacio a Madame Edwarda (1956), para nombrar solo algunos ejemplos-. Y porque conforma, si se quiere, una verdadera ontología, hace las veces de $s u$ ontología. Basta, para ir un poco más lejos y como mencionábamos más arriba, en detenerse en el prefacio a su novela Madame Edwarda -novela que le pertenece a Bataille pero que éste firma bajo el ignoto nombre de Pierre Angèlique- para ver formulada esta ontología en forma aún más explícita, es decir para leer ya del todo elaborado y del todo claro este estatuto ontológico que ocupa el exceso en su filosofía: "el ser -escribe allí Bataillenos es dado en un desbordamiento (dépassement) intolerable del ser" (Bataille 1956, p. 22. La traducción es mía). $\mathrm{O}$, mejor aún: “el exceso (excès) -escribe un poco más adelante- es aquello mismo por lo que el ser, en primer lugar, es" (Bataille 1956, p. 
31. La traducción y el resaltado son míos). Si como observábamos al principio la vida y el pensamiento de Bataille están tan estrechamente vinculados o articulados entre sí es precisamente por esto: porque Bataille, para decirlo rápidamente, no solo vivió una vida de excesos, sino porque también pensó al exceso como constitutivo de la vida, como el horizonte insondable de una ontología. Con el objeto -en suma- de situar la especificidad teórica que dicho exceso ocupa en el pensamiento del ensayista francés: la de constituir, como veremos al final del trabajo, una ontología política posmetafísica fuertemente vinculada a la práctica de la escritura, en las siguientes páginas haremos primero un breve repaso por la forma en la que el tema es abordado por Lévi-Strauss y el posestructuralismo en general, para llegar finalmente al pensamiento político posfundacional (heredero, en este sentido, del posestructuralismo), cuya vigencia y actualidad en la teoría y la filosofía política son indiscutibles.

\section{El estatuto ontológico del exceso: de Lévi-Strauss al posestructuralismo}

Lo cierto es que Bataille no fue, ni mucho menos, el único en otorgarle al exceso este estatuto ontológico y esta primacía. La reflexión a propósito del lugar ontológico del exceso cuenta con una larga tradición en el pensamiento occidental. Una tradición que incluye a Lévi-Strauss y, más ampliamente, a la disputa posterior a Lévi-Strauss, es decir a la disputa que se desprende del pensamiento de Lévi-Strauss y que incluye al estructuralismo y al posestructuralismo, y al pensamiento político posmarxista o posfundacional. En un excelente trabajo sobre Althusser, Emilio de Ípola (2007) va, incluso, más lejos y plantea que tanto el posestructuralismo como el posmarxismo posestructural no hicieron "otra cosa que repetir lo que el estructuralismo "a secas" de Lévi-Strauss había planteado (...) punto por punto y con toda claridad” (p. 107) en su texto de 1950 sobre Marcel Mauss. Comencemos, entonces, por el principio. En la Introducción a la obra de Marcel Mauss (Lévi-Strauss 1979), publicado -precisamentecomo introducción a una serie de ensayos de Mauss compilados bajo el nombre de Sociología y antropología, Lévi-Strauss se detiene en una noción a la que el propio Mauss le dedicó un parte importante de su trabajo y cuyos resultados fueron plasmados, sobre todo, en su Esbozo de una teoría general de la magia (Mauss 1979). La noción a la que hace referencia Lévi-Strauss en aquel texto es la noción de mana. El mana, describe Mauss en su ensayo, es una palabra que tiene su origen en la región de Melanesia pero que es común no solo a todas las lenguas melanesias propiamente dichas sino también a la mayoría de las lenguas polinesias. El término es estudiado por Mauss en virtud de su uso en los ritos mágicos de las comunidades primitivas que integran esta región específica de Oceanía. La singularidad que sin embargo presenta la noción de mana es que ésta no solo alude, en el contexto de estos ritos mágicos, "a una fuerza" o a "un ser" (Mauss 1979, p. 122), sino que también es utilizada como sinónimo de "una acción, una cualidad, un estado" (Ibid.). Es decir: "es a la vez-aclara Mauss- un sustantivo, un adjetivo y un verbo" (Ibid.). De una cosa, por ejemplo, se puede decir que es mana, lo que significa que tiene esa cualidad y en ese caso es una especie de adjetivo -un adjetivo que sin embargo no es posible ser usado para calificar a los hombres-. De un hombre, un ser o un espíritu, en todo caso, se puede decir que tiene 
mana: el mana de hacer esto o lo otro. Aquí, en consecuencia, el término ya no funciona como adjetivo sino como sustantivo: es algo que se tiene, una cosa, que generalmente la tienen los individuos calificados precisamente con mana-los magos- en el contexto de un rito o un acto mana. Por último, el mana puede también hacer las veces de una acción: la acción de dar mana. En cualquier caso, concluye Mauss, lo esencial es que la palabra subsume una cantidad de ideas que pueden designar diferentes cosas: "poder de brujo, cualidad mágica de una cosa, cosa mágica, ser mágico, tener poder mágico, estar encantado, actuar mágicamente" (Ibid., p. 123). El vocablo reúne así una serie de sentidos que aunque tienen un cierto parentesco entre sí no dejan de dar cuenta de una palabra que es "vaga y oscura" y al mismo tiempo "abstracta y general". De hecho, Mauss sostiene que es posible encontrar en otros lugares además de en Melanesia nociones que cumplen la misma función que el mana. Por ejemplo: entre los malayos de Detroits está el kramat: hay lugares, cosas, momentos, animales, espíritus, hombres y brujos que son kramat, que tienen kramat y que actúan con los poderes kramat. En la antigua región francesa de Indochina, los Ba-hnars expresan una idea análoga a la de mana cuando dicen que la bruja es una persona deng o que tiene deng. En Madagascar, la palabra hasina designa una cualidad sagrada que pertenece al mismo tiempo a ciertas cosas, a determinados seres, animales u hombres y especialmente a la reina. En América del Norte, otro de los lugares en donde es posible encontrar este tipo de vocablos, los iroqueses suelen utilizar la palabra orenda -aunque, sostiene Mauss, quizás los más famosos de todos estos ejemplos sean los términos que cultivaron los algonquinos y los sioux, que responden en el fondo también al mana melanesio y que son los términos manitou y wakan-. Ahora bien: en el texto que Lévi-Strauss le dedica a Mauss la lectura que hace el primero difiere en relación con la función que el segundo le otorga a estas nociones en su clásico estudio sobre la magia. En primer lugar, y como es evidente, Mauss restringe la función del mana y similares a los ritos mágicos y a la forma en la que ellas operan en el contexto de esos ritos o en los ecos que ese pensamiento mágico tiene en la vida cotidiana de una comunidad. $\mathrm{O}$, mejor aún, tanto el mana como el resto de estas nociones que mencionábamos explican y forman ese pensamiento mágico que es propio de las comunidades arcaicas. Y, en segundo lugar, y por efecto de esta misma argumentación, Mauss entiende que son nociones que se desprenden de la naturaleza arcaica de esas comunidades, por lo que solo podrían existir en este tipo de sociedades. Para Lévi-Strauss, sin embargo, el análisis de Mauss debería conducirnos a un análisis más profundo. La pregunta fundamental que se hace Lévi-Strauss es entonces si las diversas concepciones del tipo mana no están en realidad más extendidas de lo que piensa Mauss y si ellas no indican, por lo tanto, la "presencia de una forma de pensamiento universal y permanente que lejos de caracterizar determinadas civilizaciones o "estados" arcaicos o semiarcaicos de la evolución del espíritu humano" (Lévi-Strauss 1979, p. 36. El resaltado es mío) son resultado, en realidad, "de una determinada situación del espíritu al encontrarse en presencia de las cosas (de cosas extrañas o exóticas, sin explicación en lo inmediato o desconocidas: volveremos sobre esto enseguida), apareciendo, por tanto, cada vez que se produce esa situación" (Ibid.). Para apoyar esta hipótesis, Lévi-Strauss se sirve de dos ejemplos: uno que menciona el propio Mauss en su Esbozo sobre una teoría general de la magia, y otro que es producto de su propio trabajo etnográfico. El primero es el que se desprende de una observación del Padre 
Thavanet a propósito de la noción de manitou y el segundo es el que se deduce de la forma en la que un grupo de nativos de la tribu Tupi-Kawahib reaccionaron frente al regalo que los antropólogos -Lévi-Strauss incluido- les hicieron al llegar al lugar. En ambos casos se trataba de designar un objeto desconocido o poco familiar: una salamandra a la que una mujer de la comunidad de los algonquinos le tenía miedo - a la que llamaba precisamente manitou-, y un trozo de franela roja que los nativos de la tribu TupiKawahib no conocían en absoluto -y al que llamaron bicho-. Lo que, en fin, intenta demostrar Lévi-Strauss es que efectivamente las nociones de tipo mana que analiza Mauss no solo no pertenecen únicamente al pensamiento mágico y a las comunidades arcaicas sino -decíamos- a una "forma de pensamiento universal", de la que nuestras propias sociedades no están exentas, y que se ejerce frente a la presencia de objetos, situaciones o individuos que aparecen frente al observador o al actor como desconocidos, indescifrables en lo inmediato o carentes aún de nombre que los designe o identifique. En las sociedades actuales, palabras como truc o machin cumplen en la lengua francesa esta función. En francés, truc quiere decir coso, truco -lo que lo acerca en este sentido más estrechamente al sentido del mana melanesio- y suele también utilizarse para hablar de una persona desconocida -lo que en español designamos con el nombre de fulanito-. En español, en efecto, también tenemos y hacemos uso de este tipo de palabras: el término mismo coso designa por lo general algo que, o bien es desconocido, o bien lo conocemos pero no sabemos cómo nombrarlo. La palabra ángel o aura suelen también utilizarse en castellano para describir el poder o el talento -lo que vuelve a acercar a estos términos más estrechamente al uso del mana melanesio y al poder mágico que éste designa- que tiene una persona pero cuyo origen y forma son totalmente indeterminados. Es decir: para Lévi-Strauss de lo que se trata no es de suprimir o desatender el rol que este tipo de nociones tienen en la configuración del pensamiento mágico de las comunidades primitivas sino, en todo caso, de extender su función más allá de ese rol e, incluso, de tomar esa función para designar las características mágicas de determinadas cosas, seres o individuos como parte de una función en la que esa dimensión está involucrada: la de "permitir que se ejerza el pensamiento simbólico a pesar de las contradicciones que le son características" (Lévi-Strauss 1979, p. 40). Y estas contradicciones que le son características tienen -escribe Lévi-Strauss- una forma bien precisa: en "su esfuerzo de comprender el mundo -sostiene-, el hombre posee un exceso de significados". Como vemos, y para retomar el argumento que esbozábamos al principio, el análisis de Lévi-Strauss a propósito del mana y las diferentes nociones de este tipo en la obra de Mauss muestra el lugar ontológico que ocupa el exceso en todo sistema simbólico: es constitutivo, o pertenece a la dimensión del ser u ontológica, de cualquier sistema simbólico y esto es así independientemente del tipo de sistema simbólico con el que estemos confrontándonos: el de una sociedad arcaica, semiarcaica o moderna. Si el mana, el wakan, el orenda y las nociones de este tipo vienen a "permitir que se ejerza el pensamiento simbólico" es -en suma- precisamente porque permiten su sutura, son "absolutamente necesaria(s) para que en el total los significados disponibles y las cosas significadas señaladas guarden entre sí la relación de complementariedad que es condición esencial para el ejercicio del sistema simbólico" (Ibid.). Su valor "simbólico cero", su capacidad de designar todo aquello que aún no cuenta con un significado, lo desconocido e incluso lo mágico o inexplicable, hace posible así el cierre 
o la sutura del sistema, es su condición de posibilidad. Y la condición de posibilidad, va de suyo, del sentido mismo.

Ahora bien: como bien señala Emilio de Ípola -a quien retomábamos un poco más arriba- el posestructuralismo posterior a Lévi-Strauss no hizo otra cosa que repetir punto por punto y con toda claridad lo que Lévi-Strauss ya había formulado en su Introducción a la obra de Marcel Mauss: en primer lugar, la identificación de un exceso que pertenece a la dimensión ontológica de cualquier sistema o estructura o, más ampliamente, que es constitutivo de lo real, del ser o de lo que es -o, para volver sobre los términos de Bataille, lo que es, el ser, está siempre en exceso-. Aquí los nombres y las figuras que adopta este excedente ontológico son diversos y dependen de cada autor. Pero lo cierto es que desde Lacan hasta Derrida, pasando por Althusser y demás, todos ellos han debatido en torno a la reflexión sobre esta "ración suplementaria" que constituye a toda estructura o sistema simbólico, o más ampliamente, al sentido, a la verdad, al logos o a lo real mismo. Vale la pena recordar lo que el propio Emilio de Ípola destaca en su trabajo sobre Althusser para dar cuenta de esta presencia a todas luces prematura y temprana del "problema fundamental de todo estructuralismo", como señala en otro texto Badiou (1969, p. 285, nota), en el pensamiento de Lévi-Strauss y más específicamente en su texto sobre Mauss: "el problema -escribe entonces de Ípola- (...) posee una historia cuyos comienzos se remontan a cerca de una década antes de que Lacan "reconozca" lo Real y doce o trece años antes de la publicación de los primeros grandes trabajos de Althusser" (de Ípola 2007, p. 107). Si, para seguir con la argumentación de de Ípola, el texto de Lévi-Strauss es muy anterior a los textos en los que Lacan reconoce lo Real -figura que precisamente vendría a encarnar este exceso ontológico del que hablamos- es también muy anterior a los textos en donde Derrida reconoce la presencia de este mismo exceso o resto ontológico bajo las nociones de la huella (trace) o de la différance -la formulación de la primera se remonta recién a 1967 con la aparición de De la grammatologie (Derrida 1967), y la segunda a 1968 con la Conferencia que Derrida da en la Sociedad francesa de Filosofía (Derrida 1972) ${ }^{3}-$. En segundo lugar -decíamos- la apertura de este vasto espacio teórico que inaugura Lévi-Strauss coincide, o halla su continuidad con las corrientes posestructuralistas posteriores, con la identificación de un elemento o un representante (lieu-tenant) -dirá con algo de imprecisión, desde nuestro punto de vista, Badiou en su texto de los "70 que mencionábamos (Badiou 1969, p. 285, nota)-, es decir un significante, por caso, que se ocupa de realizar la operación de sutura del orden simbólico, del sistema o de la estructura, es decir, del sentido" (Miller 1988). Sabemos, en virtud de lo que venimos comentando, que ese significante, ese elemento o representante que equilibra la estructura, que la sutura, es en Lévi-Strauss el significante con valor simbólico cero, es decir el término o la palabra que señala la necesidad de un contenido simbólico suplementario al que ya tiene la cosa significada, pudiendo ocupar por eso mismo el lugar de cualquier

Una lectura algo más minuciosa de la forma en la que opera este exceso ontológico en Derrida puede verse en Mónica Cragnolini: Derrida, un pensador del resto (Cragnolini 2012). 
cosa significada: el mana, el wakan o la orenda que describe Mauss en su Ensayo sobre una teoría general de la magia, pero que en el caso de las corrientes "pos", o, más en particular, en el caso de Lacan o Derrida, para seguir con nuestros ejemplos, toman la forma de nociones tales como las de point de capiton en Lacan (Zizek 2009 y Miller 1988), o de la escritura bajo tachadura en Derrida -la escritura de la "a" reemplazando a la " $\mathrm{e}$ " en la palabra francesa différence es el ejemplo, si se quiere, más contundente- ${ }^{4}$. Un punto que, sin embargo y a pesar de esta insoslayable continuidad que une y reúne al estructuralismo con el posestructuralismo, en sus diferencias y en sus debates, que los une y reúne por lo tanto y en el sentido de que los agrupa alrededor de este problema que Lévi-Strauss formula y enuncia con toda claridad en su Introducción a la obra de Marcel Mauss, un aspecto igualmente indiscutible e insoslayable los separa: el de la posibilidad de que, con la identificación de este exceso ontológico, se abre para las teorías "pos": el de la imposibilidad final del cierre de la estructura, del sistema o del sentido, es decir, el de la inadecuación final e irreversible entre significante y significado. A diferencia del estructuralismo, entonces, el pensamiento posterior a Lévi-Strauss va hacer de esta exceso ontológico la posibilidad permanente y siempre latente, y por lo tanto nunca superable o saldable, de la subversión del sistema o de la estructura, haciendo posible, para recuperar precisamente las palabras del último Barthes, más cerca del posestructuralismo que del estructuralismo de sus inicios, "la hemorragia permanente por la que la estructura (...) se escurriría, se abriría, se perdería (...) (y) que nada puede, en definitiva, cerrar" (Barthes 2002, p. 936. La traducción es mía).

\section{El estatuto ontológico del exceso y el pensamiento político posfundacional: Rancière y Laclau}

De la amplia variante de corrientes "pos" herederas de este problema, y herederas en particular de la forma en la que éste vuelve para hacer lugar a una reflexión que se desplaza de la localización del exceso en su función de sutura del sistema o la estructura a una que enfatiza su función de subversión o de apertura (del sistema o la estructura), me interesa sobre todo una variante específica: la del pensamiento posfundacional o posmarxismo. En primer lugar, porque esta "variante" ocupa hoy un lugar decisivo en el pensamiento político contemporáneo (Marchart 2009). Y en segundo lugar -lo que en parte se desprende de esto último- porque a partir de este exceso ontológico este pensamiento traza el horizonte de lo que pensamos con el nombre de política. En la tradición de la filosofía política, para recuperar una vez más un trabajo de De Ípola (2001), la concepción de la política estuvo subsumida a dos metáforas distintas. La metáfora débil o sistémica de la política, y la metáfora fuerte o rupturista de la política. Con la primera, la política es comprendida -o reducida- a una esfera específica de lo social, a una especie de subsistema de un sistema más amplio en el que ella opera,

4 Remitimos, para una mayor comprensión de esta lógica de la escritura bajo tachadura en Derrida, al excelente trabajo de Gayari Chakravorty Spivak, titulado Sobre la deconstrucción. Introducción a De la gramatoogia de Derrida (Spivak 2013). 
aunque con sus limitaciones, y del que forma parte, que integra, y que es el sistema social. Una buena parte de la tradición del pensamiento político hizo suya esta metáfora débil de la política. Con la segunda, en cambio, la política ocupa un lugar bien distinto: encarna, ya no una esfera específica de lo social, sino el momento mismo de institución de lo social, de su apertura ${ }^{5}$. El posmarxismo o el pensamiento posfundacional se inscribe en esta segunda corriente. Es decir, adhiere a la metáfora fuerte de la política. Ahora bien: si la política es para el posfundacionalismo el momento de institución o de apertura de lo social, es porque la política excede lo social. El exceso ontológico del que venimos hablando, dicho de otro modo, es lo que hace posible a la política. Para recuperar una bellísima expresión de Martín Plot (2008), inspirada en la filosofía de Merleau-Ponty, si la política es la carne de lo social, porque la carne-escribe Plotno es "ni materialidad inerte ni etérea espiritualidad, ni pura negatividad ni pura positividad" (p. 9), materialidad por lo tanto "autoconfigurada", maleable y reversible -permitiendo esta maleabilidad y reversibilidad darle forma al cuerpo social y abriendo así la posibilidad de la configuración y reconfiguración de ese cuerpo, de su carne o de su forma- esta carne está siempre en exceso, y eso es también lo que hace posible esta maleabilidad y reversibilidad, la configuración y reconfiguración del cuerpo social ${ }^{6}$. Dos ejemplos son, en este sentido, ilustrativos de la forma en la que opera este exceso que posibilita la existencia de la política en el pensamiento posfundacional. El primero es el caso de Laclau. Laclau designa a este exceso con el nombre de "campo de la discursividad": "Este exceso - escribe el autor en Hegemonía y estrategia socialista-, en la medida en que es inherente a toda situación discursiva es el terreno necesario de constitución de toda práctica social" (Laclau 2010, p. 151). Lo que Laclau llama campo de la discursividad no es ni más ni menos que el "exceso de sentido" (Ibid.) -del que por otro lado hablaba el propio Lévi-Strauss en el texto que analizábamos más arribaque amenaza toda articulación discursiva, es decir, toda identidad política o discurso. La emergencia del pueblo solo es posible, de este modo, en la medida en la que existe siempre un excedente de sentido que no forma parte del campo de representación discursiva que configura lo social. El campo de la discursividad desborda y excede siempre al discurso en torno al cual se articula y configura la sociedad. En La razón populista, 20 años después de la publicación de Hegemonía y estrategia socialista, Laclau va a denominar a este exceso de sentido con el nombre de "heterogeneidad social" (Laclau 2008). Para resumir un poco esta idea, entonces, Laclau va a referirse a ella unas décadas después diciendo que "existe (siempre) un real del pueblo que resiste la integración simbólica” (Laclau 2008, p. 191). La política (el pueblo) se funda -en suma- en este exceso ontológico que es el del sentido, y que el autor designó primero

5 Otra forma de comprender estas dos concepciones de la política que formula de Ípola es la que desarrolla Olivier Marchart en el trabajo citado a partir de la diferencia entre la política y lo político (Marchart 2009). Puede verse también al respecto el excelente ensayo de Eduardo Rinesi: Politica y tragedia. Hamlet, entre Hobbes y Maquiavelo (2005).

$6 \quad$ Para Martin Plot, vale aclarar, es el advenimiento del régimen o el horizonte democrático o estético de la política el que hace posible esta permanente configuración y autoconfiguración de la carne de lo social (Plot 2014). 
como campo de la discursividad, y que luego reformula como heterogeneidad social. El segundo ejemplo al que hacíamos referencia es el caso de Rancière. Para Rancière, sin embargo, el exceso es el exceso que hace posible la igualdad última -o primera, en el sentido de fundamental- que permite la estructuración de cualquier orden social -a pesar de que este ordenamiento sea siempre desigualitario- (Martínez 2013): “el poder del pueblo -escribe Rancière en un artículo contra Derrida- es el exceso o el suplemento que constituye la política como tal" (Rancière 2012/3, p. 159. La traducción y el resaltado son míos). Y el poder del pueblo es el poder de la igualdad, la capacidad de confirmar la capacidad de cualquiera de gobernar y, por lo tanto, la capacidad de nadie en particular (de n'importe qui): "pero hay un solo principio -agrega Rancière en el texto que mencionábamos- (...) que queda (...) en exceso sobre todos los otros: el principio (...) o la cualificación de los que no tienen ninguna cualificación particular" (Rancière 2012/3, p. 160. La traducción y el resaltado son míos). El dèmos, en el sentido aristotélico, o la parte de los que no tienen parte (Rancière 1995) son las categorías con las que el autor francés suele referirse a los sujetos políticos que encarnan este exceso igualitario que ningún orden o sociedad puede domesticar (Rancière 2015).

\section{Bataille y la escritura: hacia una ontología politica posmetafísica}

Si volvemos al pensamiento de Bataille, vemos enseguida que este exceso ontológico es objeto de una reflexión filosófica distinta en relación con el pensamiento estructuralista, posestructuralista y posfundacional, pero de una reflexión filosófica que no deja de ser por eso una reflexión política -y esto es en parte lo que queremos mostrar: que en Bataille existe una ontología política, pero una ontología política distinta -insistimosa la del pensamiento político actual, el posfundacional, por un lado, y una ontología política que, por el otro, es posible inscribir en el marco de un régimen u horizonte posmetafísico de la política-(Martínez 2018; Plot 2018). En primer lugar, porque este exceso nada tiene que ver, en Bataille, con el sentido (como en el caso del estructuralismo o posestructuralismo posterior) o con la igualdad (como en el caso de Rancière) ${ }^{7}$. Es un exceso de energía, una energía que excede -decíamos más arriba- la energía necesaria para el mantenimiento de la vida. La vida, para Bataille, está en exceso, en exceso de energía, y a este exceso, dice por lo tanto el autor francés, hay que gastarlo (dépenser) o mejor aún dilapidarlo. La dilapidación del exceso es necesaria a la vida y no porque esa necesidad sea teleológica, sino porque es en todo caso ontológica. El gasto, como sostiene el filósofo italiano Rocco Ronchi en un artículo sobre Bataille, es "dilapidación de la sustancia" (Ronchi 2000/1, p. 110. La traducción es mía), del ser-decimos nosotros-, de la ousía. El gasto, en consecuencia, nos "acerca" en algún punto a la muerte, revela nuestra finitud, la posibilidad última de la desaparición de la vida, de la substancia de la que estamos hechos -la carne, para seguir a Plot, de nuestros cuerpos-. Por eso escribe Bataille en La part maudite, que el movimiento que él estudia es "el

Aunque, como veremos enseguida, algo de esta relación con la igualdad permanece. 
del excedente de energía, traducido en la efervescencia de la vida" (Bataille 1967b, p. 50. La traducción y el resaltado son míos). El gasto o la dilapidación es precisamente eso: el movimiento que traduce el excedente de energía en la efervescencia de la vida, en la desaparición de nuestra carne. A lo largo de su obra -y de su vida, no nos olvidemos, decíamos también más arriba, de la articulación tan estrecha en Bataille entre pensamiento y vida- Bataille encontró distintas formas de dilapidar o gastar ese excedente (Bataille 1967a). El sexo, la bebida, el juego. Y fundamentalmente la escritura. En el prólogo a La part maudite, Bataille escribe: "Escribiendo el libro en el cual yo decía que la energía finalmente debe ser gastada, empleaba yo mismo mi energía (...). Me atreveré a decir que en estas condiciones yo no podría muchas veces sino responder a la verdad de mi libro" (Bataille 1967b, p. 51. La traducción y el resaltado son míos). El vínculo de este gasto de energía, de esta dilapidación de la sustancia, de la carne de la que estamos hechos, este morirse en vida o esta traducción del gasto como forma de habitar la efervescencia de la vida, el vínculo - decíamos- de este gasto con la escritura es puesto una y otra vez en juego en la obra -y la vida- de Bataille: "esta mano que escribe es moribunda -escribe en el prefacio a Madame Edwarda-y por esta muerte a ella prometida, ella escapa a los límites aceptados escribiendo (aceptados por la mano que escribe pero rechazados por aquella que muere)" (Bataille 1956, p. 32). La escritura es así el lugar de este movimiento en el cual lo que muere acepta su muerte, la precipita dilapidando la sustancia de la que está hecha lo que muere, pero escapa a sus límites precisamente escribiendo, inscribiendo esa efervescencia de la vida en el papel que se escribe $^{8}$. Ahora bien: lo que quisiera subrayar es que esta práctica, la de la escritura, es como la de la constitución del pueblo en Laclau, como la de la emergencia de la parte de los sin parte o el demos en Rancière, una práctica política, porque es el momento de institución -digámoslo aunque sea en un primer momento muy provisoriamente- de lo social. No ya de lo social en el sentido más sociológico del término, porque configura o reconfigura el cuerpo social, su carne, sino porque instituye otra forma "de lo social", una comunidad "ni comunal ni estrictamente política" -como dirá Nancy (2016, pp. 21-22) con algo de imprecisión, como veremos enseguida- una comunidad, en fin, sin carne-para retomar a Plot y a Merleau-Ponty-, sin corporalidad, sin cuerpos presentes -plenamente- en el espacio de su aparición o configuración:-instituye -en suma- lo que en otro trabajo llamábamos la comunidad política de la escritura (Martínez 2019). Sin carne y sin corporalidad, en primer lugar, porque se trata de una comunidad que no tiene, estrictamente hablando, lugar. No encarna ni bajo la forma ni como el cuerpo del pueblo, ni bajo la figura ni como el cuerpo del demos (pero tampoco bajo la forma o el cuerpo de la Nación, de la clase proletaria o de los trabajadores). No tiene, estrictamente hablando, espacio de aparición. Es este espacio, el que se abre ahora, en el momento en el que estoy escribiendo. Es aquel, o cualquier otro espacio, que se abre cuando escribe otro. Es un no lugar, o una comunidad en la que no están presentes, plenamente,

8 En otro lugar reparamos en el hecho de que esta inscripción de la efervescencia de la vida en la escritura se produce como la inscripción del gesto singular del que escribe. Cf. Martínez (2018). 
los que la forman: ni el que lee ni el que escribe, pero que la forman la ausencia del que lee cuando se escribe, y la ausencia del que escribe cuando se lee. ¿Qué es, en fin, la comunidad de los que no tienen comunidad de la que hablaba el propio Bataille y de la que hablaron, después de Bataille, Nancy (1999) y Blanchot (1983), sino es esta comunidad sin carne, esta comunidad sin cuerpos que la constituyan, es decir la comunidad de la escritura, que es esta misma comunidad que se abre, en suma, con este texto y con esta escritura? Una comunidad de iguales, como quisiera Rancière (2007), y por eso -decíamos en parte en desacuerdo con Nancy- una comunidad política.

\section{Referencias bibliográficas}

Badiou, Alain (1969), "El (re)recomienzo del materialismo dialéctico”, en Saul Karz, ed., Lectura de Althusser; pp. 247-287. Buenos Aires: Galerna.

Barthes, Roland (2002), "Sur la lecture", Euvres complètes; tomo IV. Paris: Seuil.

Bataille, Georges (2003), La conjuración sagrada. Ensayos 1929-1939. Buenos Aires: Adriana Hidalgo Editora.

(2016), La experiencia interior. Suma ateológica I. Buenos Aires: El cuenco de Plata.

(1956), Madame Edwarda. Paris: Jean-Jacques Pauvert Éditeur.

(1967a), La notion de dépense. Paris: Minuit.

(1967b), La part maudite. Paris: Minuit.

Blanchot, Maurice (1983), La communauté inavouable. Paris: Minuit.

Derrida, Jacques (1967), De la grammatologie. Paris: Minuit.

(1972), Marges de la philosophie. Paris: Minuit.

Cragnolini, Mónica (2012), Derrida, un pensador del resto. Buenos Aires: La Cebra.

De Ípola, Emilio (2007), Althusser, el infinito adiós. Buenos Aires: Siglo XXI.

(2001), Metáforas de la política. Rosario: Homo Sapiens.

Laclau, Ernesto (2010), Hegemonía y estrategia socialista. Hacia una radicalización de la democracia. Buenos Aires: Fondo de Cultura Económica.

(2008), La razón populista. Buenos Aires: Fondo de Cultura Económica.

Lévi-Strauss (1979), "Introducción a la obra de Marcel Mauss", en Marcel Mauss, Sociología y antropología; pp. 13-42. Madrid: Tecnos.

Marchart, Olivier (2009), El pensamiento politico posfundacional. La diferencia política en Nancy, Lefort, Badiou y Laclau. Buenos Aires: Fondo de Cultura Económica.

Martínez, Juan José (2013), "Entre la (filosofía) crítica y la (filosofía de la) emancipación: el problema del orden social en el pensamiento de Jacques Rancière", Entramados y perspectivas. Revista de la Carrera de Sociología 3 (3): 191-205. 
(2019), “La communauté déplacée. Écriture et communauté dans le dialogue entre Maurice Blanchot et Jean-Luc Nancy", Agora. Papeles de filosofia 38 (2): 197-209.

(2018), Politique de l'écriture. Paris: L'Harmattan.

Mauss, Marcel (1979), Sociología y antropología. Madrid: Tecnos.

Miller, Jacques-Alain (1988), Matemas II. Buenos Aires: Manantial.

Nancy, Jean-Luc (1999), La Communauté désœuvrée. Paris: Christian Bourgois.

(2016), La comunidad revocada. Buenos Aires, Mar Dulce: 2016.

Plot, Martin (2008), La carne de lo social. Buenos Aires: Prometeo.

(2018), "Preludio: lo político y la política en Schmitt y Lefort", Unidad Sociológica 12 (3): 6-13.

(2014), The Aesthetico-political. The question of democracy in MerleauPonty, Arendt, and Rancière. Nueva York / Londres: Bloomsbury.

Pornofiello, Marisol (2017), Comunicación en Georges Bataille. (Tesis inédita de grado). Buenos Aires: Universidad de Buenos Aires.

Rancière, Jacques (2007) Aux bords du politique. Paris: Gallimard.

(2015), "La dimensión estética: estética, política, conocimiento". Ciencia Política 10 (19): 19-44.

(2012/3), "La démocratie est-elle à venir? Éthique et politique chez Derrida", Les Temps Modernes 669-670: 157-173.

(1995), La mésentente. Politique et philosophie. Paris: Galilée.

Rinesi, Eduardo (2005), Política y tragedia. Hamlet, entre Hobbes y Maquiavelo. Buenos Aires: Colihue.

Ronchi, Rocco (2000/1), “Une ontologie de l'excès”, Lignes 1: 107-124.

Spivak, Chakravorty Gayari (2013), Sobre la deconstrucción. Introducción a De la gramatología de Derrida. Buenos Aires: Hilo Rojo Editores.

Surya, Michel (2014), Georges Bataille. La muerte obra. Barcelona: Arena.

Zizek, Slavoj (2009), El sublime objeto de la ideología. Buenos Aires: Siglo XXI. 